\title{
Representaciones y significaciones del embarazo en adolescentes de 15 a 19 años en Puerto Cortés
}

\author{
Tania Waldina Puerto Zaldívar ${ }^{1}$ \\ Juan Manuel Ciudad ${ }^{2}$
}

\section{RESUMEN}

El tema del embarazo y la maternidad en la adolescencia ha sido analizado mediante diversas metodologías y enfoques teóricos, que demuestran estadísticamente la incidencia creciente de la maternidad temprana y la complejidad de su comprensión en diversos contextos socioeconómicos y culturales. Ante esta realidad, existen investigaciones más recientes en LAC, que abordan la maternidad temprana desde una propuesta transdisciplinaria, para aproximarse con mayor profundidad a la comprensión del fenómeno, partiendo de una mirada subjetiva que permita explorar el significado del embarazo y la maternidad a través del discurso, mediante el cual, las protagonistas construyen conocimiento a través de sus propias percepciones. En este sentido, a partir de los indicadores del embarazo en adolescentes en Honduras, fue pertinente realizar una investigación cualitativa con una visión más holística y ecléctica, en la cual el objetivo general es "explorar las principales representaciones y significaciones percibidas por las adolescentes embarazadas y madres en Puerto Cortés, mediante el paradigma epistemológico del construccionismo social y el sustento teórico de la Sociología Fenomenológica de A. Schütz y las Representaciones Sociales de S. Moscovici, para abordar el embarazo en adolescentes de manera más integral. Con este propósito, participaron de forma voluntaria catorce mujeres adolescentes gestantes y madres a quienes se les realizó una entrevista en profundidad que proporcionó resultados que se vinculan a subcategorías de representaciones como: postergación de los estudios, deserción escolar, identidad de género: madre-mujer-ama de casa, nuevo estatus social, maternidad esperada, proyecto fáctico, pérdida de libertad y aspiraciones educativas futuras.

Palabras clave: adolescencia, embarazo adolescente, representaciones sociales, significado de vida

\footnotetext{
${ }^{1}$ Estudiante de Sociología, Carrera de Sociología, Departamento de Ciencias Sociales, UNAH-VS: waldy.puerto@gmail.com

${ }^{2}$ Asesor, profesor de la asignatura de Taller de Investigación Sociológica II, Carrera de Sociología, UNAH-VS.: juan.ciudad@unah.edu.hn
} 


\section{ABSTRACT}

The topic of pregnancy and motherhood in adolescence has been analyzed through a variety of methodologies and theoretical approaches that statistically demonstrate the increasing incidence of early motherhood and the complexity of its understanding in diverse socioeconomic and cultural contexts. Faced with this reality, there is more recent research in LAC, which addresses early motherhood from a transdisciplinary proposal, to approach in greater depth the understanding of the phenomenon. It starts from a subjective view that allows to explore the meaning of pregnancy and motherhood through discourse, whereby the protagonists construct knowledge through their own perceptions. In this sense, from the indicators of pregnancy in adolescents in Honduras, it is pertinent to carry out a qualitative research with a more holistic and eclectic view, in which the general objective is "to explore the main representations and meanings perceived by pregnant adolescents and mothers in Puerto Cortes, through the epistemological paradigm of social constructionism and the theoretical support of the Phenomenological Sociology of A. Schütz and the Social Representations of S. Moscovici, to address pregnancy in adolescents in a more comprehensive way. For this purpose, fourteen pregnant teenage women and mothers were interviewed in depth, which provided results that are linked to subcategories of representations such as postponement of studies, school dropout, gender identity: mother-woman-home, new social status, expected maternity, factual project, loss of freedom and future educational aspirations.

Key words: adolescence, teenage pregnancy, social representations, meaning of life 


\section{INTRODUCCIÓN}

El embarazo y la maternidad en adolescentes es un tema de actualidad y de mucha importancia debido a la complejidad de sus causas como a las repercusiones que el fenómeno tiene en la vida de las mujeres adolescentes, de sus familias y de la comunidad.

En América Latina "se prevé, igualmente, que los partos en menores de 15 años se incrementen a tres millones por año en esta región para 2030." (UPNFPA, 2013, pág. 1) De este modo "La mayoría de países latinoamericanos están entre los 50 primeros del mundo en fecundidad adolescente" (Banco Mundial, 2013, pág. 1). En el caso particular de Honduras, es el segundo país con las tasas más elevadas en embarazo adolescente (26\%) (UNFPA, 2013).

De acuerdo con (ENDESA, 2012, pág. 112) el embarazo en la adolescencia tardía "el $24 \%$ de las mujeres de 15 a 19 años de edad alguna vez estuvo embarazada, bien sea porque está gestando por primera vez (5\%) o porque ya son madres (19\%)", lo cual representa un aumento de dos puntos porcentuales desde la ENDESA 2005-2006.

De este modo, la información estadística muestra que, "el porcentaje que ya son madres es bajo en las mujeres de 15 y 16 años y se incrementa rápidamente hasta llegar al 28 por ciento a los 18 años y al 36 por ciento a los 19 años" (ENDESA, 2012, pág. 112) y que esta circunstancia se vincula, con las adolescentes que se encuentran en los quintiles más pobres, ya que tienen "más de tres veces la probabilidad de haber sido madres que las adolescentes en el quintil superior (29 y 8 por ciento, respectivamente). Al igual que los embarazos entre las que poseen un nivel de escolaridad bajo: en "el primer ciclo de primaria (42 por ciento) o sin educación (35 por ciento). Por el contrario, en las mujeres con educación secundaria, el 12 por ciento han sido madres y en las mujeres con educación superior el 1 por ciento." (ENDESA, 2012, pág. 113)

La encuesta ENDESA considera un punto importante para esta investigación, el hecho de que "los embarazos a muy temprana edad forman parte del patrón cultural de algunas regiones y grupos sociales," ya que algunas mujeres jóvenes contraen matrimonio a temprana edad o se embarazan "en situaciones de unión consensual, lo que generalmente termina con el abandono de la mujer y del hijo, configurando así el problema social de la madre soltera." (ENDESA, 2012, pág. 112) 
Los departamentos que presentan mayores y menores tasas de embarazo en adolescentes en Honduras son: Gracias a Dios (35\%) e Islas de la Bahía 14\%. Cortés, no es el departamento que presenta la mayor incidencia en embarazo de adolescentes a nivel nacional, sin embargo, en Puerto Cortés, las autoridades del hospital de área de este municipio, han reportado a los medios de comunicación local y nacional la incidencia de este fenómeno con una tendencia hacia el alza en los últimos años. (ENDESA, 2012, pág. 113) Y además, que "del total de los alumbramientos, el 60 por ciento es por partos en menores de 18 años, quienes tienen incluso su segundo 0 tercer bebé antes de salir de la adolescencia."(Niñas siguen pariendo niños en el hospital, 2015)

De esta manera, de acuerdo a las proyecciones del embarazo en adolescentes en LAC, la incidencia se incrementará, por tanto, se realizan esfuerzos para una mayor comprensión del fenómeno desde una perspectiva construccionista, en investigaciones realizadas en las últimas décadas en países de LA como Chile, Colombia, Perú, Paraguay o Brasil, sobre las construcciones subjetivas del embarazo en adolescentes, como sujetos con capacidad de agencia, capaces de construir conocimiento y significado desde la cotidianidad y mediante la representación de su propia realidad, cuyos contextos económicos, sociales y culturales presentan algunas semejanzas con la incidencia del fenómeno en Honduras, lo cual ha permitido explorar las representaciones del mundo de vida construidas en esta región del país, para contribuir con los esfuerzos que se realizan en favor de la salud sexual reproductiva y la prevención del embarazo en adolescentes.

En suma, los principales resultados de las subcategorías objetivas e intersubjetivas dan cuenta de representaciones de la maternidad propias de la expresión cultural hondureña y categorizadas a partir de los hallazgos de representaciones y significaciones en investigaciones realizadas en LA, como (Agurto Galvez, 2012), (Pino, y otros, 2011), (Gomez-Sotelo, y otros, 2012), (Ovies Saavedra, 2011), (Quintero Rondon \& Rojas Betancur, 2015), entre otros.

\section{METODOLOGÍA}

Este estudio es de corte cualitativo-interpretativo, con enfoque construccionista, el nivel es exploratorio y el diseño es inductivo y emergente, con la técnica de análisis sociológico del discurso, para lo cual se realizó saturación de categorías, al crear nuevas y establecer relaciones entre las categorías existentes, a partir de las en las 
representaciones sociales percibidas y construidas por las participantes en un ambiente natural durante el proceso del embarazo y la maternidad.

La población participante en el estudio es de 14 mujeres adolescentes; siete embarazadas y siete madres, en edades comprendidas de 15 a 19 años que reciben servicios de salud en el hospital de área de Puerto Cortés y para seleccionar la muestra se verificaron los criterios de inclusión de las participantes en la investigación. La triangulación de los datos se realizó en la discusión de resultados.

\section{INSTRUMENTOS}

Guías de entrevistas semiestructuradas: Se diseñaron dos guías de entrevistas semiestructuradas, para cada grupo participante, correspondiente a las mujeres adolescentes embarazadas y mujeres adolescentes madres, con la finalidad de explorar las representaciones en ambos grupos de estudio. La guía consta de tres secciones de preguntas que incluyen los imaginarios sociales que representan las condiciones objetivas, el embarazo, la maternidad y el proyecto de vida. Previo a la realización de la entrevista se aplicaron los consentimientos informados para los padres de familia o encargados solicitando su autorización y para las adolescentes embarazadas y madres solicitando su participación en el estudio.

\section{ANÁLISIS DE RESULTADOS}

Las representaciones sociales más relevantes dan cuenta de categorías relacionadas a las condiciones objetivas, que desde la sociología fenomenológica es de gran importancia para la sociología científica: el contexto de significado objetivo, los conjuntos de significados que existen en la cultura y que son compartidos por toda la colectividad de los actores (Ritzer, 1997, págs. 280-281) , de esta forma las representaciones relevantes para los resultados de esta investigación, han sido seleccionadas de acuerdo a la frecuencia en que se presentan en el discurso de las participantes y entre las cuales resaltan: la postergación de los estudios y la deserción escolar, puesto que el $50 \%$ de las participantes manifestaban haberse retirado de la escuela antes de salir embarazadas:

"Si cuando yo tenga el bebe porque supuestamente lo voy a tener en diciembre 
entonces, el otro año que venga voy a entrar de nuevo."(C5,2:34,13:13)

"No ya no, ya no estaba en la escuela, yo cuando ya lo conocí a él ya tenía dos años de haber salido de la escuela." (C3,13:3,8:8)

Otra subcategoría representada es la identidad de género, con el ser mujer, el cual está asociado a las representaciones de la mujer como madre y ama de casa, es decir "Primero Madre y luego mujer", también a un nuevo estatus social con el coincide el $57 \%$ de las participantes y en el que las madres adolescentes se convierten en "mujeres responsables y abnegadas" dispuestas en algunos casos a cambiar hasta su forma de vestir por una apariencia más conservadora y asumir entre otras consecuencias, la perdida de libertad que conlleva la maternidad. En este mismo sentido, perciben representaciones culturales-religiosas que exaltan la figura de "Dios otorgador de maternidad" y por tanto, la preponderancia de las RS de la madre, sobre la mujer.

"Mujer, bueno tengo muchos aspectos, ahora que soy mamá, he dejado eso de ser, como le dicen mujer, porque siempre me han dicho que, primero se es mamá que ser mujer." (C7, 11:33,20:20)

En cuanto a los deseos de estar embarazadas se pudo constatar, a nivel exploratorio en las RS de pensar y sentir la maternidad que la mayoría de las adolescentes participantes en el estudio, es decir el $64 \%$ de ellas, desean tener un hijo, como una decisión trascendental que transformará sus vidas, en sus propias palabras:

"No, yo siempre he deseado tener un niño" (C6, 3:11,32:32)

P: "No, fue decisión de los dos. E: ¿Fue decisión de los dos?, o sea ustedes estuvieron de acuerdo los dos que querían tener un bebé. P: (asintió con la cabeza.)" (C13, 14:13,38:38)

Es importante mencionar que, para fortalecer la percepción del embarazo deseado, se analizaron los contenidos discursivos de las respuestas obtenidas mediante la técnica expresiva de asociación libre, utilizando fotografías con imágenes de adolescentes en distintos momentos del embarazo, y se tomaron los comentarios de la imagen número uno, que representa la reacción de la mujer adolescente al enterarse que está embarazada y se compararon con las respuestas brindadas por las participantes al preguntársele si su embarazo era esperado o inesperado. 
"No, fue una sorpresa, porque yo sí tenía, no me cuidaba, porque ya había tenido como seis meses de estar con el papa de la niña y no salía embarazada y de repente....jsalí embarazada!". (Rostro de sorpresa y alegría) (C2, 8:38, 38:38)

"Sobre la prueba del embarazo yo me la hice de sangre ....cuando yo miré, justamente, cuando otra muchacha parece que estaba esperando, y la doctora dijo las dos salieron positivas, cuando yo escuche eso, pues, digo yo así, va, como el positivo de cualquier otra cosa, pero yo cuando miré el papel ¡me alegró! porque yo quería tener a alguien a mi lado con quien yo platicar, porque con mi mami si platicaba conmigo, pero yo no tenía como alguien pues, con quien estar ahí siempre, mi hermano iba por un lado, mi mami por otro, y entonces, yo no tenía alguien, con quien estar siempre juntos, ni con quien dormir, pero ahora ya tengo a mi niña."(C2IM1, 8:24,71:71)

De este modo, la noticia del embarazo desencadena reacciones variadas, pero con mayor frecuencia de sorpresa, de alegría, y evidencia las carencias afectivas en el entorno familiar, que están representadas en el deseo de tener un hijo, que se convierte en el centro de su vida, en una serie de contenedor emocional que ha venido al mundo para complementar sus carencias afectivas.

Respecto al significado de la maternidad para las mujeres adolescentes, se encuentran más representaciones que indican que ser madre joven es: ser valiente, responsable, luchadora y protectora de sus hijos, ellas se auto representan como madres con un gran reto y muchas ansias de demostrar que son mujeres capaces de sacar adelante a sus hijos, ellas manifiestan que aunque son dependientes de sus padres en algunos casos, comprenden que su nuevo rol materno demanda un cambio de actitudes y de esta forma dan respuesta a las críticas que surgen en las interacciones con el entorno de pares o familiares.

"Un reto, es un reto porque para mi edad me faltan muchas metas para cumplir, que no he terminado de cumplir, que me faltan, eh no tengo digamos, yo en lo personal, yo me considero de que no tengo una posición para pensar en que colegio voy a poner a mi hija cuando este grande, no, no trabajo, solamente dependo de mis padres, de que si ellos no me quieran dar pues, yo tengo que ver como los hago, porque ahora en los tiempos que estamos ese dinerito no le va a caer del cielo."(C1,13:35,50:50)

Respecto al apoyo que las participantes reciben por parte de sus parejas, ellas representan la maternidad, como un proyecto que se anticipó con el embarazo o la llegada 
del bebé, sus representaciones son muy variadas respecto a las actitudes que asume su pareja frente a la maternidad, identificando al "cariñoso y responsable" como las categorías predominantes:

P: "Me trata bien "E: ¿Y cómo es el? ¿le ayuda con el bebe? P: Sí, el me ayuda, cuando lo escucha llorar, me ayuda también, él se desvela E: Ah se desvela con usted, ¿Y él trabaja? P: Si E: y ¿él tiene la misma edad que usted o es mayor? P: No, diecinueve años tiene, E:... ¿Y él vive aquí? P: Si aquí vive. (C14, $14: 22,65: 65)$

Otros códigos se asocian con: la inmadurez la irresponsabilidad, la indiferencia, la manipulación de la pareja y las relaciones contradictorias, ya que en algunos casos, el padre del bebé inicia siendo muy afectivo y luego realiza amenazas y acciones violentas:

"Él fue una persona de que siempre me dio mi lugar sea como sea, él es ya muy súper mayor que mí, él tiene ya 27 años, estamos hablando de diez años de diferencia, él siempre me dijo: no usted es la que manda, lo que usted decida, ino el hombre es el que manda a la mujer, no la mujer al hombre!, eso siempre lo he tenido bien claro...Él ahora tiene una orden de alejamiento, y decidí ponerle una orden de alejamiento, porque él estaba allí, como yo no lo dejaba ver, yo no lo dejo ver a la niña, entonces, él estaba ahí como poniéndome amenazas, si no me enseñas a la niña, o no me dejas ver a mi hija te voy a matar. Entonces, tengo que ver por mi vida, por la de mi familia y por la de mi hija, entonces, no digo yo vamos a hacerlo por la ley, ahí si él intenta romper la ley, pues. (Cl1,13:23,68:68)

En el caso de C11, ella contaba con el apoyo de su familia y pudo superar esta situación de violencia doméstica, en la cual, como manifiesta la participante existen relaciones de poder de una pareja mayor. Las adolescentes que no cuentan con el respaldo de sus padres o la confianza para comunicarles sus problemas, se exponen a vivir en peligrosos círculos de violencia y muerte, que en los casos más afortunados finaliza con la ruptura de las relaciones de pareja.

Las relaciones de pareja con el nacimiento del bebé también fueron reflexionadas por las participantes mediante la fotografía, entre las representaciones asociadas al nacimiento del bebé sobresalen las interacciones madre e hijo o hija, en el contexto cotidiano y que se vinculan al aprendizaje de las madres adolescentes en el cuidado de sus bebes: 
"si, yo cuando tuve la niña solo pendiente de ella, de que no, no estuviera mal. Ahí con ella cambiándola a cada rato, La bañaba con cuidadito porque me daba miedo primero, Pero un día que estaba algo molesta así, agarré yo, la niña, digo yo voy a hacerlo, digo y ...yo solita bañe a la niña, ahí con cuidado pero, la bañé. Pero siempre hay veces, me da un poquito de miedo, porque ella cuando uno la está bañando, se mueve mucho y se desliza a veces y me da miedo golpearla. (C2,IM5, 8:51,83:83)

Otra reflexión sobre la maternidad adolescente, fue construida por una de las participantes luego de observar la fotografía: "una niña chineando a otro niño, se ve como muñequito, una niña cuidando un niño, depende de la madurez de la muchacha."($\mathrm{Cl}, \mathrm{IM} 5,1: 28,86: 86)$. Esta reflexión permite captar el salto de etapa que experimentan las adolescentes que son madres a temprana edad y también pone de manifiesto que han sido pocas las participantes que interpretan y construyen reflexiones acerca de las consecuencias del embarazo al saltarse la etapa de la adolescencia.

En referencia al proyecto de vida, las representaciones también permiten captar los significados que se interpretan como intenciones que requieren de acciones futuras para ser concretadas, pero que se construyen en el mundo de los sueños y aspiraciones, como manifiestan las participantes su deseo de convertirse en profesionales.

"pues...la verdad...yo... quiero ser doctora."(C8, 12:45,53:53)

"Yo quiero llegar a graduarme ser alguien que, una como persona ejecutiva. Pues, bueno, yo quisiera seguir estudiando, o sea, salir de estos dos niños y....que continuar con ellos, y sacar mis estudios" (C4,8:44,53:53)

Retomando a Schütz, "Y más explícitamente, Natanson señala «En cada caso, el rasgo fundamental de la acción es el hecho de ser proyectada y estar dotada de propósito". (Ritzer, 1997, pág. 281) Es precisamente el propósito lo más relevante de esta representación, ya que aunque el contexto es desfavorable existe la intención de realizar éstas y otras aspiraciones que forman parte de los imaginarios sociales representados en este estudio.

\section{DISCUSIÓN}

Partiendo del objetivo general de este estudio que es explorar las principales representaciones y significaciones del embarazo en adolescentes de 15 a 19 años en el 
hospital de área de Puerto Cortés, indican que los resultados presentan concordancias importantes en comparación con los hallazgos de otras investigaciones vinculadas al mismo tema en la región latinoamericana, razón por la cual, se adoptaron varias subcategorías para clasificar las representaciones sociales resultantes del estudio realizado en este municipio del país.

\section{Tabla 1. Principales representaciones sociales del embarazo y la maternidad en adolescentes de 15 a 19 años en Puerto Cortés}

\begin{tabular}{|c|c|c|c|}
\hline Sub-categoría & Embarazadas & $\begin{array}{l}\text { Madres } \\
\text { (primigestantes y } \\
\text { multigestantes) }\end{array}$ & Convergencias \\
\hline $\begin{array}{l}\text { Vinculación } \\
\text { Educativa }\end{array}$ & $\begin{array}{l}\text { Postergación de los } \\
\text { estudios y deserción } \\
\text { escolar, desatención de } \\
\text { los padres, apoyo } \\
\text { educativo pertinente. }\end{array}$ & $\begin{array}{l}\text { Postergación de los } \\
\text { estudios por maternidad, } \\
\text { deserción escolar por } \\
\text { frustración, continúan } \\
\text { los estudios }\end{array}$ & $\begin{array}{l}\text { Postergación de los } \\
\text { estudios y deserción por } \\
\text { bajos ingresos }\end{array}$ \\
\hline $\begin{array}{l}\text { Auto representación } \\
\text { de la Maternidad } \\
\text { (se vincula al ser } \\
\text { mujer) }\end{array}$ & $\begin{array}{l}\text { Ser Mujer: madre-ama de } \\
\text { casa, no sabe que es ser } \\
\text { mujer }\end{array}$ & $\begin{array}{l}\text { Mujer: madre-ama de } \\
\text { casa, es bonito ser } \\
\text { mujer y madre. }\end{array}$ & $\begin{array}{l}\text { Mujer: madre ama de } \\
\text { casa, es difícil por el } \\
\text { machismo. }\end{array}$ \\
\hline $\begin{array}{l}\text { 1. RS embarazo } \\
\text { adolescente } \\
\text { maternidad } \\
\text { 2. consecuencias }\end{array}$ & $\begin{array}{l}\text { Nuevo status social: } \\
\text { primero madre luego } \\
\text { mujer, el hijo como única } \\
\text { propiedad. }\end{array}$ & $\begin{array}{l}\text { Nuevo status social: } \\
\text { abnegada }\end{array}$ & $\begin{array}{l}\text { 1. Responsable, } \\
\text { usa vestimenta } \\
\text { conservadora. } \\
\text { 2. Perdida de libertad }\end{array}$ \\
\hline $\begin{array}{l}\text { Percepción de } \\
\text { cambios positivos y } \\
\text { negativos atribuidos } \\
\text { a la maternidad y } \\
\text { situación actual. }\end{array}$ & $\begin{array}{l}\text { Rechazo social y familiar: } \\
\text { cometió un error, críticas } \\
\text { de otras mujeres. }\end{array}$ & $\begin{array}{l}\text { Rechazo social y } \\
\text { familiar: lo tomaron con } \\
\text { normalidad, expulsión } \\
\text { del hogar. }\end{array}$ & Enojo de los padres \\
\hline $\begin{array}{l}\text { Experiencia del } \\
\text { embarazo desde la } \\
\text { propia perspectiva. }\end{array}$ & $\begin{array}{l}\text { Embarazo inesperado: } \\
\text { temor, sorpresa }\end{array}$ & $\begin{array}{l}\text { Embarazo inesperado: } \\
\text { asustada }\end{array}$ & $\begin{array}{l}\text { Embarazo deseado: } \\
\text { sorpresa, alegría }\end{array}$ \\
\hline $\begin{array}{l}\text { Consecuencias } \\
\text { actuales de } \\
\text { 1. En la pareja } \\
\text { 2. Nacimiento del } \\
\text { bebe }\end{array}$ & $\begin{array}{l}\text { 1.Indiferente, manipulador } \\
\text { 2. interacción con el bebe, } \\
\text { reflexiona la maternidad, } \\
\text { impresionada. }\end{array}$ & $\begin{array}{l}\text { 1.Responsable, } \\
\text { Inmaduro, } \\
\text { irresponsable, } \\
\text { contradictorio } \\
\text { 2. aprendizaje e } \\
\text { interacción con el bebe: } \\
\text { regalo de Dios, } \\
\text { prepararse para criar los } \\
\text { hijos. }\end{array}$ & $\begin{array}{l}\text { 1.Consentidor, cariñoso } \\
\text { 2. interacción con el } \\
\text { bebé }\end{array}$ \\
\hline $\begin{array}{l}\text { Proyecciones } \\
\text { educacionales y } \\
\text { laborales } \\
\text { (proyecto de vida } \\
\text { emergente) } \\
\end{array}$ & $\begin{array}{l}\text { Estudiar para trabajar, } \\
\text { proyecto fáctico, ser } \\
\text { profesional }\end{array}$ & $\begin{array}{l}\text { Trabajar para demostrar } \\
\text { que puede, Ser } \\
\text { profesional universitario, } \\
\text { ningún proyecto de vida. }\end{array}$ & $\begin{array}{l}\text { Estudiar para el } \\
\text { bienestar de los hijos, } \\
\text { ser profesional }\end{array}$ \\
\hline
\end{tabular}

Fuente: elaboración propia a partir de los resultados obtenidos en las Representaciones y Significaciones del Embarazo y la Maternidad en Adolescentes de 15 a 19 años en Puerto Cortes y algunos elementos del formato tomados de (Gomez-Sotelo, y otros, 2012) 
De este modo, comenzando con el plano contextual del análisis del discurso, en el cual se analiza la realidad objetiva de las participantes que optaron por el proyecto fáctico de la maternidad temprana, esta realidad se vincula a la pobreza, la desintegración familiar, la deserción escolar y la postergación de los estudios.

Respecto al proyecto fáctico, en el estudio de (Quintero Rondon \& Rojas Betancur, 2015, pág. 229) sus resultados revelan que el proyecto de vida es sustituido por el denominado "proyecto fáctico, el cual, "tiene que ver con las implicaciones de los embarazos y la maternidad a temprana edad", es decir que existe un salto de etapa, desde la adolescencia hacia la adultez prematura mediante la maternidad temprana. Como resultado "Las mujeres adolescentes afirmaron que aunque el estudio es prioritario, ya habían abandonado sus estudios antes de salir embarazadas." A este respecto otra investigación realizada por Emma Naslund y Georgina Windstock, en Perú y Paraguay, revela que "Se ha encontrado que el embarazo de adolescentes en LAC está asociado con la pobreza (Chedraui y otros 2004, Florez 2005, Guijarro y otros 1999, Guzmán y otros 2001, Pantelides 2004, Peña y otros 1999, Porras 2003), con bajos logros en la educación (Alcázar y Lovatón 2006, Giovagnoli y Vezza 2009, Pantelides 2004, Ríos-Neto y Miranda-Ribbeiro 2009)," etc. (Naslund Hadley \& Binstock, 2011, pág. 2)

En este mismo sentido, un estudio sobre representaciones y significaciones como (Pino, Escobar, Muñoz, Torrent, Bosch, \& Barreto, 2011, págs. 122-123) realizado en Chile, respecto a la vinculación educacional, resaltaba la falta de apoyo para el cuidado del hijo y como resultado la deserción escolar (femenina).", al igual que en Gomez Sotelo y Otros 2012, entre las RS exclusivas del embarazo y la maternidad en adolescentes convergentes en ambas poblaciones (primigestantes y multigestantes) se encuentran ligadas a la postergación de los estudios, la dependencia familiar y económica, entre otras RS, que están ligadas a la deserción escolar. Por tanto, es importante mencionar que los hallazgos recientes sugieren que:

"Más de la mitad de las madres adolescentes paraguayas y cerca de un cuarto de las peruanas habían abandonado la escuela antes de su embarazo. La razón principal que adujeron para descontinuar sus estudios fue la falta de interés que había generado bajos niveles de logro y la repetición de uno o más grados." (Naslund Hadley \& Binstock, 2011, pág. 12)

Respecto a la maternidad temprana vinculada al plano intersubjetivo las RS que dan cuenta de la identidad de género, es decir, ser mujer, a partir del ser madre, la maternidad significa un nuevo status social que brinda sentido y reconocimiento a las 
labores cotidianas en el ámbito familiar, ya que la joven madre se auto representa como responsable y protectora de su única propiedad, un nuevo ser que la convierte en madre, por tanto mujer y ama de casa. En este sentido, en (Gomez-Sotelo, y otros, 2012) se constata la importancia del enfoque constructivista, Algunos hallazgos de RS en (primigestantes y multigestantes) están vinculados a un nuevo estatus social. Por otro lado la presencia de un nuevo ser, se vincula al proyecto de vida pues, despierta en los padres adolescentes según Pino y Otros 2011, "un deseo de estudiar y prepararse con el objetivo de brindarle a sus hijos calidad de vida." (Pino, Escobar, Muñoz, Torrent, Bosch, \& Barreto, 2011, pág. 125) Además en "Los hallazgos sugieren que las adolescentes que enfrentan obstáculos que desalientan el aprovechamiento académico y las aspiraciones altas en la vida tienen también muchas probabilidades de quedar embarazadas." (Naslund Hadley \& Binstock, 2011)

De este modo, la afirmación de desear tener un hijo, por parte de la mayoría de las adolescentes embarazadas, adquiere significaciones subjetivas asociadas a la identidad de género, a carencias afectivas, educativas y económicas vinculadas al contexto familiar en el que han crecido y a la ausencia de un proyecto de vida personal que ha sido sustituido por un proyecto fáctico, que representa un salto de la niñez a la adultez.

"Algunas participantes llegaron al punto de planear sus embarazos, como dijo una mujer que tuvo su primer hijo a los 16 años: "Tomé precauciones el primer mes. Después dejé de hacerlo. Tenía esa idea loca de que debía tener un bebé" (Naslund Hadley \& Binstock, 2011, pág. 13).

La maternidad esperada por mujeres en adolescencia temprana se evidencia en (Agurto Galvez, 2012, pues, "Llama la atención que una de las entrevistadas manifiesta el querer y haber deseado tener un hijo, aun cuando tenía 14 años de edad. Lo indica como una posibilidad para llenar sus vacíos y aquellas carencias afectivas" (Agurto Galvez, 2012, pág. 72). Por tanto en el proceso del embarazo, es vital el apoyo familiar:

"Una vez que comienzan a sentir su hijo/a en su vientre, y ya en su gran mayoría sintiendo el apoyo de sus familias respectivas, el embarazo comienza a apreciar de otra manera, de apoco comienza a darse una mayor aceptación y van adaptándose a la llegada de éste hijo" (Agurto Galvez, 2012, pág. 72).

Caso contrario el rechazo de los padres, les expone a vivir en contextos de violencia y marginalidad. 
De este modo, la asignatura pendiente es la construcción interactiva de una identidad de género en el mundo cotidiano en el que las RS son productoras de sentido de forma simultánea, intersubjetiva y por tanto, contribuyen en la deconstrucción de patrones culturales patriarcales arraigados en la sociedad y reproducidos históricamente. Es importante resaltar que no obstante, las dificultades que deben enfrentar y las pocas oportunidades que existen en el entorno, las madres a temprana edad, tienen aspiraciones y se proyectan como proveedoras de una mejor calidad de vida para sus hijos.

\section{CONCLUSIÓN}

En concordancia con las principales representaciones sociales del embarazo y la maternidad adolescente construidas por las participantes en este estudio, se concluye que la comprensión de este fenómeno es compleja y demanda que las intervenciones que se realicen en Honduras para prevenirlo deben incluir también la exploración de representaciones y sus significados que se construyen en contextos de pobreza, desintegración familiar y bajas expectativas de logros académicos. Asimismo, es de suma importancia la construcción de la identidad del género, que es percibida y significada a partir de la maternidad y que representa la adquisición de un nuevo estatus social reivindicativo de la mujer. Por tanto, la maternidad esperada a edad temprana se vincula a carencias afectivas, educativas y económicas del entorno familiar, en el cual, las participantes perciben que la maternidad hace a una mujer diferente, responsable y que con el apoyo adecuado son capaces de concretar un proyecto de vida familiar, aunque los contextos de desarrollo sean mayoritariamente desfavorables.

\section{AGRADECIMIENTOS}

Se agradece la asesoría técnica recibida del tutor de la Tesis Lic. Juan Manuel Ciudad Joya, así como las recomendaciones de otros maestros asesores y el apoyo del personal del hospital de área de Puerto Cortés, y de la clínica del adolescente y por supuesto agradecimientos especiales a las mujeres adolescentes que abrieron las puertas de sus hogares y de sus vidas, y cuya participación hizo posible la realización de esta investigación. 


\section{REFERENCIAS BIBLIOGRÁFICAS}

Agurto Galvez, G. A. (2012). Construcción Subjetiva de Madres Adolescentes acerca de su Maternidad y Proyectos de Vida, Residentes Vulnerables de la Comuna de Cauquenes. Tesis doctoral, Universidad de Bio Bio, Departamento de Ciencias Sociales, Concepcion, Chile.URL:http://cybertesis.ubiobio.cl/tesis/2012/agurto_g/doc/agurto_g.pdf

ENDESA. (2012). Encuesta de Salud . Tegucigalpa. URL:http://www.ine.gob.hn/images/Productos\%20ine/endesa/ENDESA\%202011-2012\%20cap4.pdf.

Gomez-Sotelo, A., Gutierrez-Malaver, M. E., Issedin Bouquet, R., Sanchez-Martínez, L. M., Herrera-Medina, N. E., Ballesteros-Cabrera, y otros. (2012). Representaciones sociales del embarazo. Revista de Salud Pública , 14, 189-199. URL: http://www.redalyc.org/articulo.oa?id=42225409001

Naslund Hadley, E., \& Binstock, G. (2011). El Fracaso Educativo: embarazo para no ir a clase. Nota Técnica, Banco Interamericano de Desarrollo BID, Division de Educación. URL:https://publications.iadb.org/bitstream/handle/11319/4912/El\%20fracas0\%20educativo\% $3 \mathrm{a} \% 20$

Quintero Rondon, A. P., \& Rojas Betancur, H. M. (2015). El embarazo a temprana edad, un análisis desde la perspectiva de madres adolescentes. Revista Virtual Universidad Católica del Norte (44), 16. URL:http://revistavirtual.ucn.edu.co/index.php/RevistaUCN/article/viewFile/626/1161

Salinas Mulder, S., Castro Mantilla, M. D., \& Fernandez Ovando, C. (2014). Vivencias y Relatos sobre el embarazo en la adolescencia: una aproximacion a factores sociales, culturales y emocionales: un estudio en 6 paises de la región. Panamá. URL:http://www.unicef.org/ecuador/embarazo_adolescente_5_0_(2).pdf

Yanez, D. N. (2012). Acercamientos Teóricos a la Maternidad Adolescente como Experiencia Subjetiva. (U. A. Mexico, Ed.) REDALYC, 27 (77), 235-266. URL: http://www.scielo.org.mx/scielo.php?script=sci_arttext\&pid=S0187-01732012000300007 